\title{
Flexgrid Technologies Enabling BRAS Centralization in MANs
}

\author{
Michela Svaluto Moreolo, Josep M. Fabrega, Laura Martín, Kostas Christodoulopoulos, \\ Emmanouel Varvarigos, and Juan Pedro Fernández-Palacios
}

\begin{abstract}
In an evolutionary metro-regional area network (MAN) scenario, targeting the centralization of the broadband remote access server (BRAS), flexgrid technologies can be suitably tailored to be applied. We investigate their applicability, proposing network architecture and data plane solutions to improve the spectrum and network efficiency, while reducing CapEx investment. We first describe the MAN scenario evolution and the envisioned solution based on flexgrid for supporting BRAS centralization. Then, we define the network design problem, propose a solution, and use that to design a realistic regional network (region-A of Telefonica Spanish network), identifying the transmission requirements for such a representative case. Subsequently, flexgrid data plane solutions are presented, focusing on the design, numerical validation and experimental assessment of a sliceable bandwidth variable transponder (S-BVT) based on OFDM. We propose an architecture able to meet the identified requirements, supporting flexgrid connections of up to $50 \mathrm{~Gb} / \mathrm{s}$ per flow over a 6-hop path of $545 \mathrm{~km}$. Furthermore, an alternative scheme for the uplink is given and experimentally demonstrated in a 4-node photonic mesh network. Finally, the techno-economic viability of the proposed solution is evaluated in comparison with current practice solutions.
\end{abstract}

Index Terms - MAN, BRAS, flexgrid, elastic optical networks, sliceable bandwidth variable transponder (S-BVT), OFDM.

\section{INTRODUCTION}

$\mathbf{E}$ lastic optical networks (EONs) and flexgrid technologies open up a new paradigm for the network architecture design and require the identification of suitable data plane enablers to obtain improved spectral efficiency, increased system capacity, and network costs reduction. Under these common drivers, new advanced features are envisioned for optical transponders and nodes able to support the evolutionary approach and scenarios of future networks and

M. Svaluto Moreolo, J. M. Fabrega, and L. Martín are with the Centre Tecnològic de Telecomunicacions de Catalunya (CTTC), Castelldefels 08860, Spain (michela.svaluto@cttc.es).

K. Christodoulopoulos and E. Varvarigos are with the University of Patras, Patras, Greece.

J. P. Fernández-Palacios is with Telefónica I+D, Madrid, Spain. services [1-5]. Particularly, innovative solutions of the different elements composing next generation optical networks exploit the flexgrid granularity, using $12.5 \mathrm{GHz}$ (or even $6.25 \mathrm{GHz}$ ) spectrum slots, to improve the efficient utilization of the optical spectrum, and also the presence of superchannels for high-rate connections [1, 6].

Flexgrid (elastic) technologies have been mainly studied for core network scenarios. However, they are also considered as candidate solution for the metro-regional area network (MAN) segment, due to the high bandwidth pressure, even for next generation access networks. The reduction of channel width enables the creation of low bit rate connections, which may be used in MANs, leading to an evolutionary approach for this network segment [7]. In addition, main network operators have been recently expanding their photonic mesh to the regional networks. In this context, it has been proposed to extend the aggregation network reach, typically confined in a metropolitan area, and centralize the IP edge functionality, enabled by the broadband remote access server (BRAS). A BRAS (also referred to as B-RAS or BBRAS) routes traffic to/from access nodes such as optical line terminals (OLTs) in the Telco (telecommunications) network of an operator. The BRASes, located at the edge of the IP core, aggregate user sessions from the access network. Thus, the creation of a suitably dimensioned pool of virtual BRASes, co-located with IP transit routers in central locations, allows significant reductions in Capital Expenditure (CapEx) investment.

In an evolutionary scenario, where the BRAS capability is centralized, a flexgrid regional photonic mesh network could conveniently provide connections between several multitenant units (MTUs) and the central pool of BRASes, by exploiting the advanced features of new transmission technologies such as flexgrid transponders. Particularly, a sliceable bandwidth variable transponder (S-BVT) is able to slice the transmission to concurrently serve multiple destinations through the individual control of its carriers [4]. This feature is well aligned with the point-to-multipoint communication required for the BRASes centralization use case. However, flexgrid S-BVTs for core networks are not appropriate to support multiple low bit rate connections over regional optical paths; whereas a more cost-effective solution must be investigated.

In this paper, we analyze the applicability of flexgrid technologies in this evolutionary MAN scenario, proposing suitable network architecture and data plane solutions. In Sec. II, we describe the MAN scenario and the envisioned flexgrid solution for supporting BRAS centralization. In Sec. 
III, we define the regional network design problem and identify the transmission requirements for a representative case study. Sec. IV presents the data plane solutions, particularly focusing on the S-BVT design, providing a transceiver architecture able to meet the requirements identified in Sec. III. The numerical validation is performed taking into account the specifications and topology of a realistic regional network (namely, Region-A of the Telefonica Spanish network) and the experimental assessment is carried out in the photonic mesh network of the ADRENALINE testbed. In Sec. V the techno-economic analysis is given. Finally, conclusions are drawn in Sec. VI.

\section{Evolution of Metro Network ARchitecture}

Metro architectures in the past were in most cases composed of two levels of aggregation: (i) The first level (named multitenant user or MTU) collects traffic from the end users (optical line terminals or OLTs), while (ii) the second level (named Access) aggregates the traffic from the MTUs mainly through direct fiber connections (i.e. dark fiber). The IP functionality, including traffic classification, routing, authentication, etc., is implemented in the BRASes usually located after the second level of aggregation.

Currently, network operators are considering the virtualization of network elements such as customer premises equipment (CPE), BRAS and content delivery network (CDN) and the co-location of these elements in centralized data centers according to the network function virtualization (NFV) paradigm [8]. These Telco data centers will concentrate the IP traffic of all access nodes in wide regions (more than 1 million users). A complete network transformation is required to enable cost per bit optimization among access nodes (e.g., OLTs) and data centers hosting virtualized BRAS and CDNs. In the centralized scenario assuming a WDM regional optical network, the optical network provides the transport capacity to connect the second aggregation level to the remote BRAS (Fig. 1a).

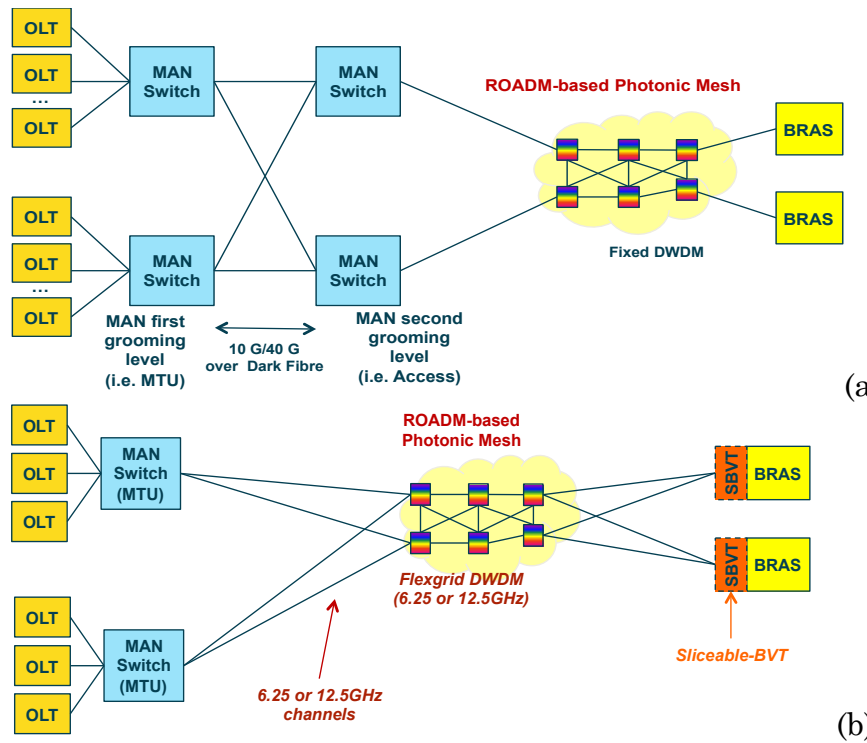

Fig. 1 (a) WDM network approach, (b) proposed architecture utilizing flexgrid and S-BVTs to interconnect BRAS with the first level of aggregation - the MTU switches.
The appearance of flexgrid transponders is paving the path for such network transformation towards cost effective optical metro-regional networks [4]. In this study, we propose the use of flexgrid technology and exploit the finer spectrum granularity $(12.5 \mathrm{GHz}$ or $6.25 \mathrm{GHz}$ slots) to remove the second aggregation level (Access). Moreover, we propose to use appropriate data plane solutions that are less complex and much cheaper than flexgrid transponders that would be used in core long-haul networks. The network architecture proposed is shown in Fig. 1b. In this evolutionary scenario, the BRAS is responsible for operating as an edge (border) node. We assume optical protection support (e.g. subnetwork connection protection, SNCP) in the photonic mesh, so that each MTU is connected via two node-disjoint lightpaths to two central BRAS. The flexgrid photonic mesh network can benefit from the use of S-BVT at the BRAS servers as it is able to generate/receive an aggregated flow of several channels, with e.g. $10 \mathrm{~Gb} / \mathrm{s}$ capacity each (MTU channels). In addition, the S-BVT has the capability of slicing an aggregated flow into multiple flows of variable capacity to be transmitted to different destination nodes. In order to handle and fully exploit sliceability and multi-flow operation of the S-BVT located at the BRAS node, OTN (optical transport network) layer is key to adapt service information content (e.g. coming from IP layer) to the photonic layer, according to the node and network target requirements $[3,4]$.

The study (detailed in the subsequent sections) highlights that the transmission distances involved do not necessarily require high performance coherent solutions. Thus, an improved S-BVT architecture based on orthogonal frequency division multiplexing (OFDM) with less complex transmission and detection schemes is proposed. A technoeconomic comparison between the WDM and flexgrid solutions shows reasonable costs for the S-BVT adopting simplified direct detection (DD) to give cost benefits of $30 \%$. Additional benefits would come from reduced flexgrid WSS costs, which dominate the cost of the solution.

\section{NETWORK DESIGN PROBLEM}

We consider a regional optical network consisting of reconfigurable add/drop multiplexer (ROADM) nodes (fixedgrid WDM or flexgrid) and fiber links. We assume that the ROADMs are colorless and directionless, but not contentionless, which is typical for current regional networks (as the one studied in Fig. 3). The network supports the traffic generated by a set of MTU switches that are lower in the hierarchy and are connected to the regional optical network. Each MTU switch is connected for protection to two different ROADMs. The way an MTU switch is connected to the two ROADMs changes according to the network scenarios (in the WDM scenario it is connected through two Access switches while in the flexgrid scenario directly to the two ROADMs). In turn, the two ROADMs, source and chosen backup source, are connected via two node-disjoint lightpaths to two BRAS nodes, for protection purposes (we assume optical protection, such as SNCP, for both fixed-grid WDM and flexgrid). There are two design problem variations: (P1) the location of the BRAS nodes is given and the goal is to minimize the maximum 
length of all primary and backup paths (min-max path length problem), and (P2) the maximum reach is given (constrained by the transponders used) and the goal is to minimize the number of BRAS locations (min number of nodes problem). Wavelength/slot utilization should also be accounted for, since the spectrum left can be used to serve other type of traffic over the same network.

\section{A. Problem definition}

The network design problem is formally defined as follows. The regional optical mesh network is represented by $G=(V, E)$, where $V$ is the set of ROADM nodes and $E$ is the set of single-fiber links, while two opposite such links are used to connect two nodes. We assume to know the link lengths, and denote by $d l$ the length of link $l \in E$. We assume the general case where an MTU switch is connected to the ROADM node $n \in V$ (this is the original location of the switch) and for backup it can be connected to one from a set $B S_{n}$ of backup ROADMs sources, $\left|B S_{n}\right| \geq 1$. Note that the case where we are given a single backup source is a subcase, and that we assume that all MTU switches connected to ROADM $n$ have the same set of backup sources $B S_{n}$. From the source and the chosen backup source, we establish two node-disjoint lightpaths to two BRAS nodes where the BRAS servers are located. We will call these ROADMs the "BRAS nodes" and denote their set by $D \subseteq V$, where $|D| \geq 2$ for fault-tolerance (the case where we have exactly 2 BRAS nodes is a subcase). All connections are of rate $C \mathrm{~Gb} / \mathrm{s}$. Fig. 2 a shows the problem.

To solve this problem, we transform it to a node-disjoint routing problem. We create an expanded graph $G^{\prime}=\left(V^{\prime}, E^{\prime}\right)$ that we initialize equal to the regional network graph $G$. We

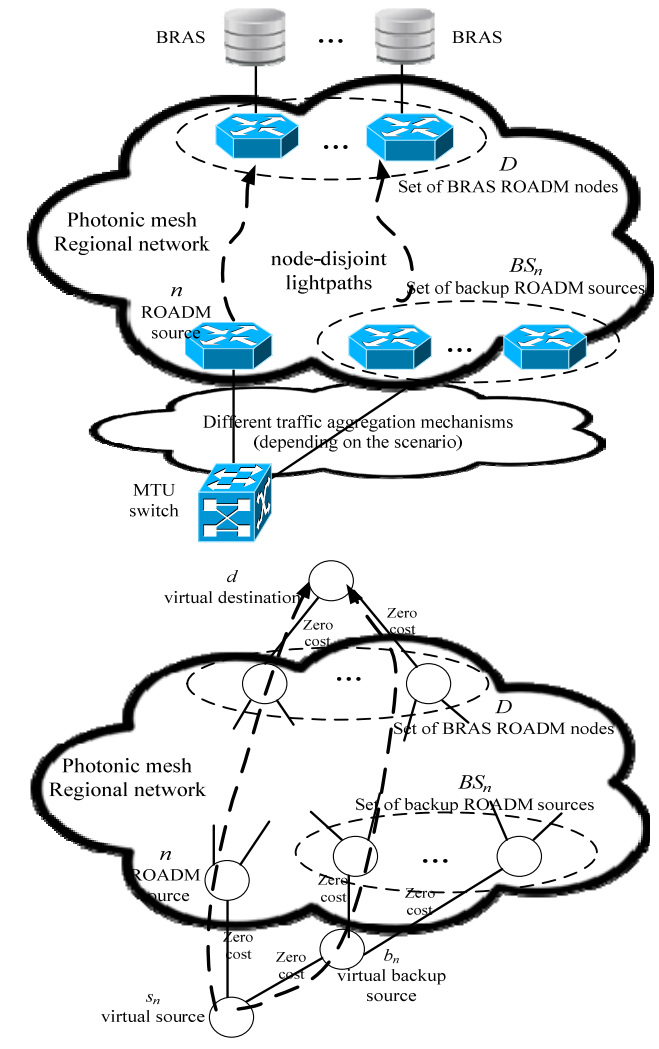

Fig. 2 (a) Network design problem, (b) equivalent node-disjoint routing problem. make the following additions to $G$ '. We create three virtual nodes: (i) the virtual MTU switch source $s_{n}$, (ii) the virtual backup source $b_{n}$, and (iii) the virtual destination $d$. The virtual MTU source $s_{n}$ is connected to the source ROADM node $n$ and the virtual backup source $b_{n}$ with zero cost links. The virtual backup source $b_{n}$ is connected to each ROADM node in the set $B S_{n}$ of backup sources and to the virtual MTU source $s_{n}$ with zero cost links. The virtual destination $d$ is connected to each BRAS node in the set $D$ with zero cost links. The cost of the links of the regional optical mesh network is taken to be their length.

The problem of selecting the backup source, the two BRAS nodes, and finding node-disjoint lightpaths is now transformed to finding two disjoint paths from virtual source $S_{n}$ to virtual destination $d$ over the extended graph $G$. This equivalent problem is shown in Fig. 2b. Based on the above transformation, which was described for a single MTU switch, we formulate the combinatorial problem to optimize the design of the whole network. We create for all MTU switches the corresponding virtual source and backup source, while the virtual destination $d$ is created once since it is the same for all MTU switches.

In the following we give a generic integer linear programming (ILP) formulation that can be used to solve both problem variations ( $\mathrm{P} 1$ and $\mathrm{P} 2)$ and can be extended to cover other cases as well. Then we focus on each variation and discuss its particularities.

\section{ILP formulation}

We assume that we are given the network graph $G=(V, E)$, the set $W$ of wavelengths (or slots) supported and the set $D$ of BRAS nodes. We are also given the number of primary connections $R_{n}$ to be established for node $n \in V$ (each requiring one wavelength/one slot), which depends on the number of MTU switches under $n$. We construct the expanded graph $G^{\prime}=\left(V^{\prime}, E^{\prime}\right)$, where $V^{\prime}$ is the set of expanded nodes and $E^{\prime}$ is the set of expanded links, as discussed above. We denote by $(m+)$ and $\left(m^{-}\right)$the set of (directed) links that start from and end at node $m \in V$, respectively.

ILP variables

$x_{\ln W}$ : Boolean variable equal to 1 if link $l$ is used by the primary path from virtual source node $s_{n}$ to the virtual destination, and equal to 0 otherwise

ylnw: Boolean variable equal to 1 if link $l$ is used by the backup path from virtual source node $S_{n}$ to the virtual destination, and equal to 0 otherwise

$z$ : Integer variable representing the highest slot utilized $u i$ : Boolean variable, defined only for links ending at virtual destination node $d$, that is, for $l_{\epsilon}\left(d^{-}\right)$, equal to 1 if link $l$ is utilized or 0 otherwise

$h$ : the longest path length of all primary and backup paths

- Flow conservation of primary \& backup paths constraints

$$
\begin{aligned}
& \forall n \in V: \sum_{l \in E^{\prime} \& l \in\left(s_{n}+\right)} \sum_{w} x_{l n w}=R_{n}, \sum_{l \in E^{\prime} \& l \in\left(s_{n}+\right)} \sum_{w} y_{l n w}=R_{n} \\
& \forall n \in V: \sum_{l \in E^{\prime} \& l \in(d-)} \sum_{w} x_{l n w}=R_{n}, \sum_{l \in E^{\prime} \& l \in(d-)} \sum_{w} y_{l n w}=R_{n} \\
& \forall n \in V, m \in V^{\prime}-\left\{S_{n}, d\right\}, w \in W: \sum_{l \in E \& l \in(m+)} x_{l n w}=\sum_{l \in E \& l \in(m-)} x_{l n w}
\end{aligned}
$$


- Node disjoint paths constraints

$\forall n \in V, m \in V^{\prime}-\left\{s_{n}, d\right\}, w \in W: \sum_{l \in E \& l \in(m-)} \sum_{w}\left(x_{l n w}+y_{l m w}\right) \leq 1$

- Worst path length constraints

$\forall n \in V: h \leq \sum_{l \in E^{\prime}} \sum_{w} d_{l} \cdot x_{l n w}, h \leq \sum_{l \in E^{\prime}} \sum_{w} d_{l} \cdot y_{l n w}$

- Wavelength (slot) allocation constraints

$\forall l \in E^{\prime}, w \in W:$

$\sum_{n \in V}\left(x_{l n w}+y_{l n w}\right) \leq 1, \quad \sum_{n \in V} w \cdot\left(x_{l n w}+y_{l n w}\right) \leq z$

- BRAS location constraints

$\forall l \in(d-), n \in V: \sum_{w \in W}\left(x_{l n w}+y_{l n w}\right) \leq u_{l}$

Note that the ROADM requirement of not being connectionless is satisfied by enforcing the single wavelength allocation constraint (6) to be applied to the virtual links $l \in\left(d^{-}\right)$and $l \in\left(s_{n}+\right)$.

\section{1) Design variation P1: min-max path length}

In problem variation $\mathrm{P} 1$ we assume that we are given location of the BRAS nodes and we minimize the longest path. This can be formulated with the above ILP with the following objective:

\section{Minimize $h+A \cdot z$}

where $A$ denotes the weigh accounting for the contribution of the highest wavelength/slot utilized in the optimization problem. In formulation for P1 we can remove Eq. (7), since the location of BRASes is given. Note that the problem at hand is NP-complete, due to wavelength assignment problem (which is NP-complete even in trees and rings of trees [19]). Assuming that we do not optimize the use of wavelengths (weight $A=0$ ), then the remaining problem of minimizing the maximum reach can be solved in polynomial time by finding for each virtual source the shortest divers paths [20]. Since ILP formulations do not scale well for large problem instances, we also developed a heuristic algorithm. The heuristic works as follows. For each virtual source $s_{n}$, we compute shortest diverse paths to destination $d$ according to [20]. We then serve nodes according to highest demand $\left(R_{n}\right)$ first, assigning wavelengths according to the first wavelength utilization policy.

\section{2) Design variation P2: Minimizing BRAS nodes}

We now consider the case where the location of the BRAS nodes can be decided by the network planner and is therefore part of the optimization problem. Instead, the maximum transmission reach of the transponders $H$ is given, and used as a constraint with the goal of minimizing the number of BRAS nodes. To optimally solve P2, we use the above ILP with the following objective:

$$
\text { Minimize } \sum_{l \in(d-)} u_{l}+A \cdot z
$$

We also add the following constraint for the reach:

$$
h \leq H \text {. }
$$

We also developed a heuristic algorithm which works as follows. For each combination of 2-BRAS nodes, we run heuristic P1. If we find a combination of 2-BRAS nodes whose min-max length is lower than the given reach $H$ we select that placement. If no 2-BRAS nodes combination is successful, we keep the best combination (defined as the one that satisfies for the given reach the most nodes) and extend that by searching for one additional node. The same is repeated at each step, adding one BRAS to the set, until all demands are satisfied with acceptable reach connections.

\section{B. Case study}

We now present a case study for the design of a realistic regional network with centralized BRAS. The goal of this study is to obtain transmission requirements and guide the design of appropriate transponders (discussed in the next Sections). In this sense the most interesting outcome of this study is the maximum length calculated in design problem variation $\mathrm{P} 1$, where we are given the placement of the BRAS nodes and we minimize the maximum length. In addition to that, results are presented for P2, so as to obtain a wider understanding of the design problem at hand.

The network studied is the region-A of Telefonica Spanish network with 30 optical nodes (Fig. 3), while traffic is generated by 200 MTU switches. We report results for the ILP formulations (using weight $A=10$ ) and for the proposed heuristics. From Table 1, we can see that for problem variation P1 with $D=\{21,22\}$ the worst case path for the BRAS placement is $545 \mathrm{~km}$ and traverses 6 nodes (path: 12-7-9-10-13). This was found both by the ILP and by the heuristic, with the heuristic using one additional wavelength. The results for problem variation P2 show that, as expected, the lower the feasible reach is, the higher the number of BRAS nodes is required. The heuristic is quite efficient, being able to find solution with the same number of BRAS nodes as the ILP for all cases apart from the case of $250 \mathrm{~km}$ reach, where it employed one more BRAS node. For high reach, which requires a low number of BRAS nodes, we can even search among all BRAS node combinations. Instead of that, the heuristic searches for an increasing number of BRAS nodes, keeping a good previous solution up to that point. The choices made are efficient in the beginning, but when many nodes are required they might be sub-optimal. All node combinations are examined by the optimal ILP, whose running time was observed to be low in these experiments, but is bound to face scalability

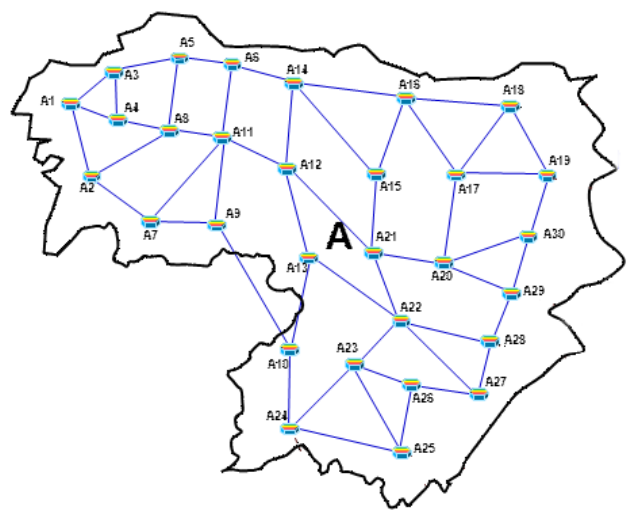

Fig. 3 TID region-A topology. 
problems in larger network and low reach scenarios.

\section{Flexible Data Plane Solutions}

A cost-effective data plane solution, tailored for the envisioned MAN scenario, is studied in this section.

It is assumed that the traffic demand per each MTU (aggregating traffic from lower level OLTs) is $C=10 \mathrm{~Gb} / \mathrm{s}$ and that traffic at the BRAS nodes is served by S-BVTs. As shown in Fig. 4, the S-BVT at the BRAS node consists of a set of virtual transceivers (TRX) serving multiple MTUs located at different nodes of the network. The aggregated flow of high capacity can be opportunely sliced into multiple flows of lower capacity routed over different lightpaths.

For BRAS centralization in a regional network, the SBVT must be both cost-effective and robust against transmission impairments, in order to support multiple low bit rate connections over optical paths of hundreds of $\mathrm{km}$.

OFDM arises as a suitable technology for rate and distance adaptive transmission in flexgrid MAN. In fact, thanks to the subwavelength granularity of OFDM, the rate can be adjusted to the request with fine granularity by using bit loading (BL) and power loading (PL). In addition, according to the channel condition, subcarriers with low signal to noise ratio (SNR) are assigned to support data mapped with the suitable modulation format to improve the robustness against transmission impairments [5].

OFDM systems using DD, thanks to their simplicity at both the transmitter and receiver sides, are promising candidates for cost-effective solutions. DD can be combined with double side band (DSB) modulation to further simplify the transponder design. Nevertheless, DSB OFDM transmission limits the achievable reach, due to the power fading induced by the chromatic dispersion (CD). Thus, to cope with the targeted optical path lengths, a possible solution is to reduce the spectral occupancy of the optical OFDM signal and thus mitigate the self-cancellation effect between the subcarriers at the two sidebands of the optical carrier [7]. Alternatively, for covering longer elastic optical paths, single sideband (SSB) can be adopted enabling enhanced robustness to $\mathrm{CD}$. This implementation requires adding an optical filter at the transmitter. In DD-OFDM systems a guard band between the optical carrier and the OFDM signal is required, to avoid the mixing products generated at the photodetector [9]. The spectral efficiency can be enhanced by minimizing the guard band and using higher size constellations in the subcarrier loading. While

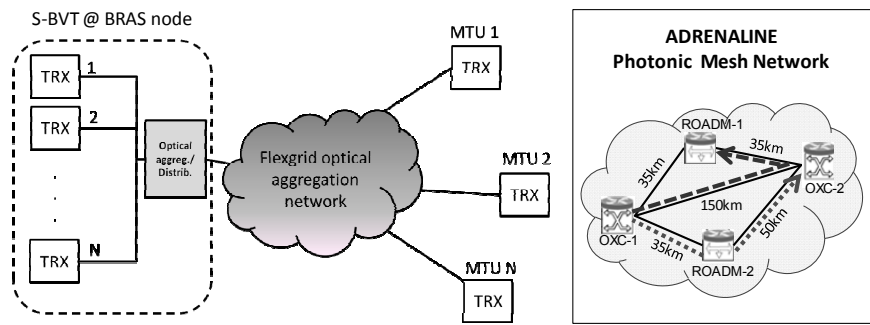

Fig. 4 Data plane solution based on S-BVT at BRAS node and ADRENALINE photonic mesh network.

minimizing the guard band, the design should take into account the suitable working point of the optical modulator and the trade-off for adopting low-cost laser (e.g. with linewidth of the order of $\mathrm{MHz}$ ).

In the following subsections, we provide data plane alternative proposals for the transceiver architecture design, targeting the BRAS centralization. The validation/assessment of the proposed solutions has been performed in the 4-node photonic mesh network of the ADRENALINE testbed, which is represented in Fig. 4.

\section{A. S-BVT architecture based on OFDM technology}

The proposed cost-effective software-defined S-BVT is able to serve $N x M$ MTUs. The transmitter array shown in Fig. 5 generates $N$ flows (slices) that can be directed towards different destinations. The bandwidth and bit rate of each flow can be varied at the digital signal processing (DSP) by software, selecting the suitable modulation format and number of active OFDM subcarriers. Multiple formats per each subcarrier are supported. The wavelength of the flexgrid channel is set at the tunable laser source (TLS).

In order to limit the array dimension and the number of optoelectronic blocks, each S-BVT array element serves $M$ MTUs with a single optoelectronic front-end. It uses only one laser source at the transmitter side (BVTx) and simple/cost-effective DD at the receiver side (BVRx). The SBVT building block is based on multi-band OFDM (MBOFDM) also referred as subcarrier multiplexing. The MBOFDM RF BVT generates an electrical signal consisting of multiple OFDM bands at intermediate radio frequency $(\mathrm{RF})$, driving the Mach-Zehnder modulator (MZM).

For covering the worst optical path, according to the analyzed regional topology and traffic, DD is combined with SSB modulation. The required guard band $\left(B_{G}\right)$ is minimized and the mixing between the optical carrier and the OFDM signal is reduced by properly adjusting the MZM

Table 1. Design results for Region-A TID reference network.

\begin{tabular}{|c|c|c|c|c|c|c|c|c|c|c|c|}
\hline & & \multicolumn{5}{|c|}{ Heuristic } & \multicolumn{5}{|c|}{ ILP } \\
\hline $\begin{array}{l}\text { Problem } \\
\text { variation }\end{array}$ & Input & $\begin{array}{l}\text { Worst } \\
\text { path } \\
\text { length }\end{array}$ & Worst path & $\begin{array}{l}\text { Time } \\
(\mathrm{sec})\end{array}$ & $\begin{array}{l}\text { BRAS } \\
\text { nodes }\end{array}$ & $\begin{array}{c}\text { Max } \\
\text { wave- } \\
\text { length }\end{array}$ & $\begin{array}{l}\text { Worst } \\
\text { path } \\
\text { length }\end{array}$ & Worst path & $\begin{array}{l}\text { Time } \\
(\mathrm{sec})\end{array}$ & $\begin{array}{l}\text { BRAS } \\
\text { nodes }\end{array}$ & $\begin{array}{l}\text { Max } \\
\text { wave- } \\
\text { length }\end{array}$ \\
\hline $\mathrm{P} 1$ & $\begin{array}{c}D= \\
\{21,22\}\end{array}$ & 545 & $1-2-7-9-10-13-22$ & 0.9 & $\{21,22\}$ & 28 & 545 & $1-2-7-9-10-13-22$ & 4.5 & $\{21,22\}$ & 29 \\
\hline $\mathrm{P} 2$ & $\begin{array}{l}\text { Reach }= \\
500 \mathrm{~km}\end{array}$ & 485 & $\begin{array}{c}3-5-6-14-12- \\
21-22\end{array}$ & 8 & $\{22,13\}$ & 32 & 500 & $\begin{array}{c}19-30-29-28- \\
22-13\end{array}$ & 27 & $\{13,21\}$ & 30 \\
\hline $\mathrm{P} 2$ & $\begin{array}{l}\text { Reach }= \\
400 \mathrm{~km}\end{array}$ & 385 & $\begin{array}{c}\text { 30-19-17-16- } \\
15\end{array}$ & 12 & $\{9,12,15\}$ & 27 & 395 & $\begin{array}{c}\text { 14-15-16-17- } \\
19\end{array}$ & 550 & $\{9,12,19\}$ & 27 \\
\hline $\mathrm{P} 2$ & $\begin{array}{l}\text { Reach }= \\
300 \mathrm{~km}\end{array}$ & 290 & $4-8-11-12$ & 20 & $\{6,12,21,29\}$ & 25 & 295 & $23-22-28-29$ & 650 & $\{6,8,21,29\}$ & 22 \\
\hline $\mathrm{P} 2$ & $\begin{array}{l}\text { Reach }= \\
250 \mathrm{~km}\end{array}$ & 240 & $4-3-5-6$ & 30 & $\begin{array}{c}\{21,17,8, \\
6,9,23\}\end{array}$ & 20 & 250 & $3-5-6-11$ & 600 & $\begin{array}{c}\{2,11,17,21 \\
25\}\end{array}$ & 21 \\
\hline
\end{tabular}


biasing point [10]. The optical SSB filter at the transmitter is included within the optical aggregator functionalities. In fact, the high capacity flow aggregation is performed by a bandwidth variable wavelength selective switch (BV-WSS or WSS), acting as both tunable filter bank and coupler.

As shown in Fig. 5, the MTU channels can be formed by a variable number $(L \geq M)$ of sub-bands $\left(B_{S k}\right)$ at variable rate, enabling distance-adaptive transmission. By considering narrow sub-bands, the flexibility and spectral efficiency can be enhanced (reducing $B_{G}$ ), and the DSP requirements relaxed. A proof of this concept has been performed up to $L=14$ sub-bands supporting $M=2$ MTU connections at $10 \mathrm{~Gb} / \mathrm{s}$ net bit rate, fitting within a single $12.5 \mathrm{GHz}$ flexgrid channel [10]. Successful sliceable transmission has been also proven over 2 -hop paths of $85 \mathrm{~km}$ and $185 \mathrm{~km}$ of the ADRENALINE network (as indicated in Fig. 4), using components with $10 \mathrm{GHz}$ bandwidth. If the bandwidth of the DAC and the optoelectronic devices is doubled, up to 5 or 6 MTUs can be served by a single MB-OFDM BVT. Thus, an S-BVT with 17 or 20 blocks could provide a total capacity of $1 \mathrm{~Tb} / \mathrm{s}$, for serving up to $100 \mathrm{MTUs}$. Thanks to its scalability, modularity, wide range granularity and capability to be programmed, the S-BVT architecture based on multicarrier technology enables smooth upgrade/adaptation to higher traffic density [3, 4]. This can be achieved either increasing the slice or band number as well as improving the DSP modules to be adaptive according to the traffic demand [5].

\section{B. Multi-band DD-OFDM analysis}

In this subsection, a more detailed analysis of the multiband system, supporting the connectivity between the SBVT located at the BRAS with the MTUs, is provided. Particularly, the assessment of an S-BVT building block serving $M=5$ MTUs is targeted.

Different implementation schemes can be used to generate the multiple bands either in the optical or in the electrical domain [11], [12]. Each implementation presents pros and cons, which should be carefully studied for the transponder design according to the specific application, considering feasibility, technological limitations and cost.

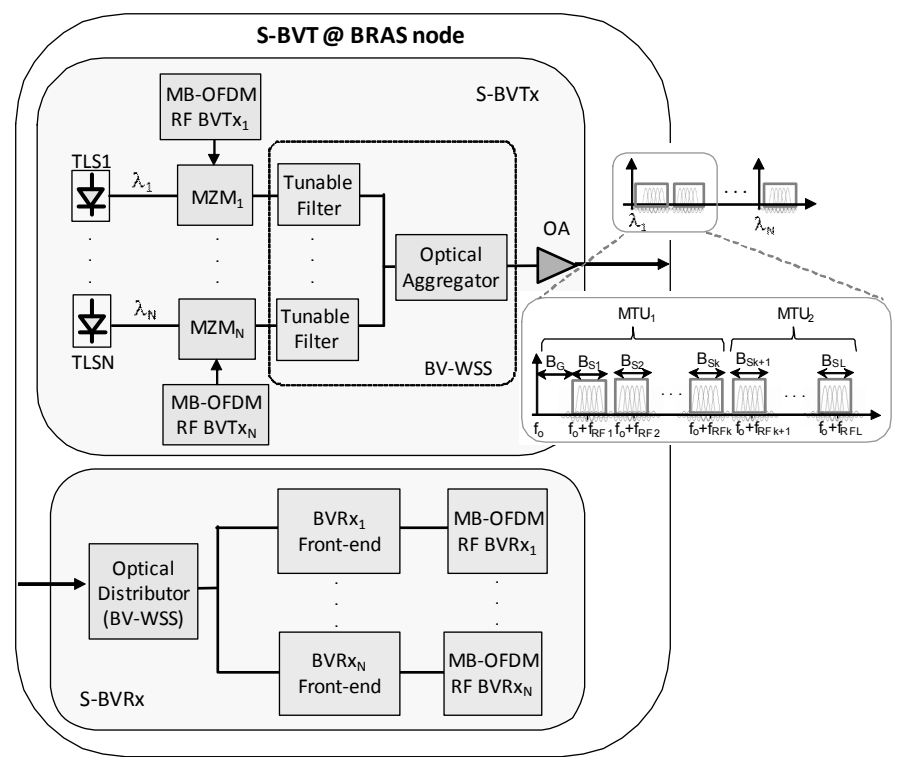

Fig. 5 S-BVT sited at the BRAS node
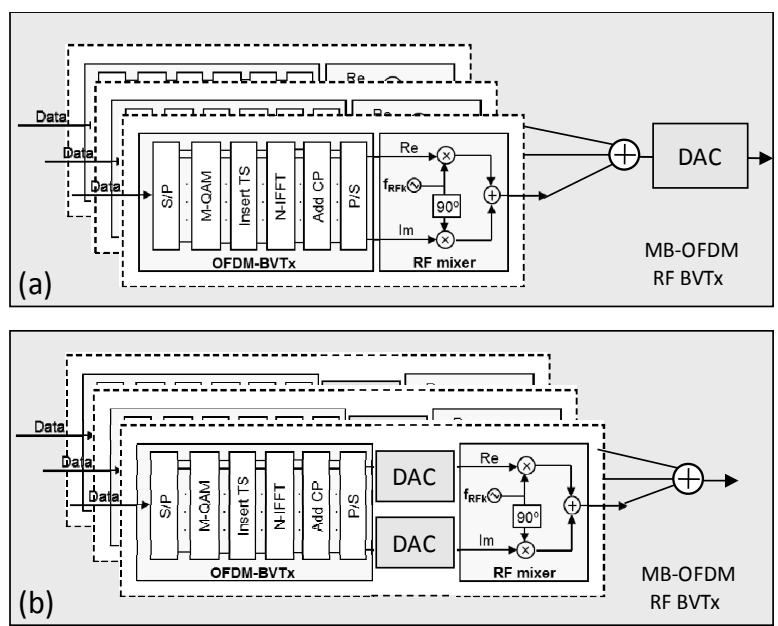

Fig. 6 MB-OFDM RF BVTx implementations with (a) digital (software-defined) and (b) electrical (hardware-based) RF mixing.

Especially for cost-sensitive applications, a critical issue to take into account is the digital-to-analog conversion. It is related to in-phase and quadrature (I/Q) mixing, total number of bands, required bandwidth and sample rate. The I/Q mixing domain can be digital, electrical or optical; for simple DD schemes, it is preferably to adopt digital or electrical mixing. The former is software-defined; the latter is hardware-based. The number $(M)$ of MTUs per MBOFDM slice (corresponding to a single optical carrier $\lambda_{n}$ ) is limited by DAC bandwidth and optoelectronic components.

In this analysis, we assume $L=M$ (the number of subbands coincides with the number of bands) and we consider components of $10 \mathrm{GHz}$ bandwidth for assuming the use of low-cost devices. Thus, the proposed MB-OFDM scheme uses electrical I/Q mixers, as shown in Fig. 6(b). At the transmitter, the input data is converted from serial to parallel. Then, this information is mapped onto a complex ( $m$-QAM) constellation. The resulting signal is processed by the inverse Fast Fourier transform (IFFT) block. Training symbols (TS) are added for data-aided synchronization and equalization at the receiver side. In order to avoid intersymbol interference (ISI) and intercarrier interference (ICI), a cyclic prefix (CP) is appended to the OFDM frames. The resulting OFDM symbol is serialized. Then, the digital signal is clipped for PAPR (peak-to-average power ratio) reduction [13], and converted to analog by means of a DAC. The same processing is applied to each band of the MBOFDM system. The next step is the up-conversion (mixing) to an intermediate frequency (RF), properly selected for each band to avoid overlaps. Therefore, this implementation uses a number of mixers equals to the number of bands. Subsequently, bands are combined in the electrical domain.

Alternatively, if the mixing is performed in the digital domain, a single higher speed $\mathrm{DAC}$ is required to convert to analog the large bandwidth MB-OFDM signal. Digital mixing enables a software-defined tuning of the electrical (sub-)bands over the spectrum, enhancing the system flexibility/tune-ability without requiring any electronic hardware. State of the art DACs at ultra-high speed allow converting signals with quite large bandwidth, featuring sampling rates up to $92 \mathrm{GSa} / \mathrm{s}$. 
A conventional MZM is used for optical modulation. The MZM creates a DSB signal with respect to the optical carrier. In order to mitigate $\mathrm{CD}$ induced power fading, one of the two sidebands is suppressed by a SSB filter [9]. This function is enabled by the WSS at the BRAS S-BVTx. Then, the resulting signal is transmitted in the network (Fig. 7).

At the receiver side, a simple photodetector is required. Afterwards, each band is downconverted to the proper intermediate frequency. The resulting signal of each band is digitized, filtered and parallelized. In case of digital mixing, the down-conversion and ADC modules are in reverse order. Finally, the signal is post-processed to recover the original bitstream: the $\mathrm{CP}$ is removed, the FFT is implemented, the equalization is performed and the signal is demapped.

The proposed MB-OFDM is simulated using Python software. We consider five bands. The total number of bits is 65536. We have analyzed the case of transmitting a net bit rate of $10 \mathrm{~Gb} / \mathrm{s}$ for each band $(50 \mathrm{~Gb} / \mathrm{s}$ for $M=5$ ) using 4 -QAM format. The number of FFT subcarriers is 512 and the total number of frames is 128 . The target BER is set to $2 \cdot 10^{-2}$, assuming an overhead of $20 \%$ due to the soft-decision forward error correction (SD-FEC). Thus, to support a net bit rate of $10 \mathrm{~Gb} / \mathrm{s}$, the bandwidth of each OFDM band is $6.11 \mathrm{GHz}$, also accounting for TS and CP. The guard band between the optical carrier and the OFDM signal equals the bandwidth occupancy of the first band $(6.11 \mathrm{GHz})$; while the guard band between two consecutive bands is $0.2 \mathrm{GHz}$. The system relevant parameters are shown in Table 2.
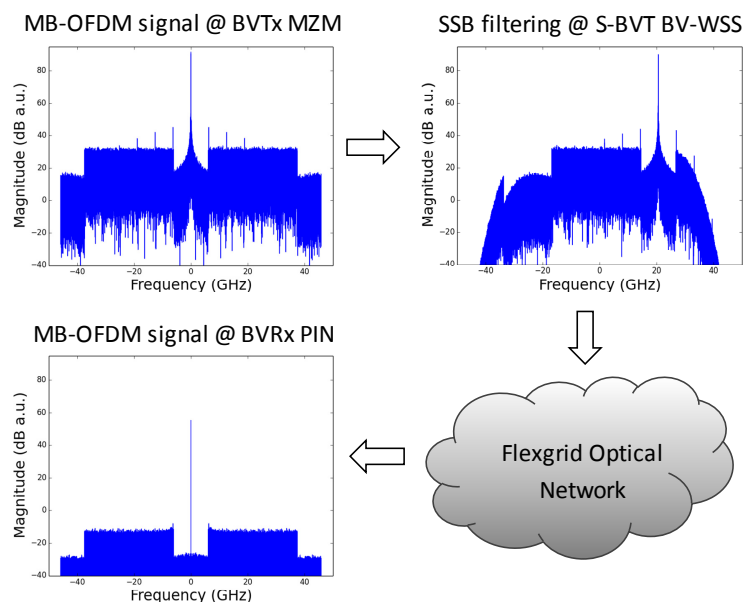

Fig. 7 Following arrows flow: DSB MB-OFDM signal after optical modulation (MZM), SSB filtering at BV-WSS and photodetected spectrum at receiver.
Table 2 MB-OFDM system parameters

\begin{tabular}{|l|l|}
\hline CP & $1.9 \%$ \\
\hline TS & $2.34 \%$ \\
\hline FEC & $20 \%$ \\
\hline Band number $(L=M)$ & 5 \\
\hline Single band occupancy & $6.11 \mathrm{GHz}$ \\
\hline Guard band & $6.11 \mathrm{GHz}$ \\
\hline Multi-band guard band & $0.2 \mathrm{GHz}$ \\
\hline Clipping factor & 3 \\
\hline Laser center wavelength & $1550 \mathrm{~nm}$ \\
\hline Laser linewidth & $1 \mathrm{MHz}$ \\
\hline MZM bias & $0.5 \mathrm{~V}$ п \\
\hline PIN responsivity & $0.7 \mathrm{~A} / \mathrm{W}$ \\
\hline Fiber type & $\mathrm{SMF}$ \\
\hline Dark current & $10^{-9} \mathrm{~A}$ \\
\hline
\end{tabular}

The total optical bandwidth occupation can be calculated as follows: $B_{T}=\sum_{k=1}^{M} B_{S k}+B_{G}+\sum_{k=1}^{M-1} B_{G k}$, where $B_{S k}$ indicates the bandwidth of the $k$-th (sub)-band, $B_{G}$ is the guard band between the first band and the optical carrier, and $B_{G k}$ is the guard band between the two consecutive bands $k$ and $k+1$. Thus, considering five bands $(M=5)$, each with the same bandwidth occupation $\left(B_{S k}=6.11 \mathrm{GHz}\right)$, including the guard bands $\left(B_{G}=B_{S k}\right.$ and $B_{G k}=0.2 \mathrm{GHz}$ ), the total bandwidth $B_{T}$ is $37.5 \mathrm{GHz}$.

Depending on the channel condition and selected path, higher modulation format can be selected according to the SNR profile, leading to narrower bandwidth occupancy. Individual subcarrier bit and power loading can be performed for optimal spectral usage and performance improvement, if specialized DSP modules are included in the S-BVT architecture [14]. The BER performance has been analyzed for different optical paths. The considered path lengths cover up to 6 hops, traversing the nodes 1, 2, 7, 9, 10, 13 and 22, for a maximum length of $545 \mathrm{~km}$. The hops and path lengths have been selected according to the worst path calculated in Sec. III.B, considering the specifications of the TID network in region-A (see Fig. 3). At the receiver, the photodetector can be modeled as a square law detector. As a result, the photodetected signal is affected by secondorder nonlinear effects [9]. These effects appear mainly at the guard band and within the first band (the closest to the optical carrier). Biasing the MZM at the quadrature point allows limiting the mixing products [7]. Furthermore, the path length also affects the performance of the system. As a
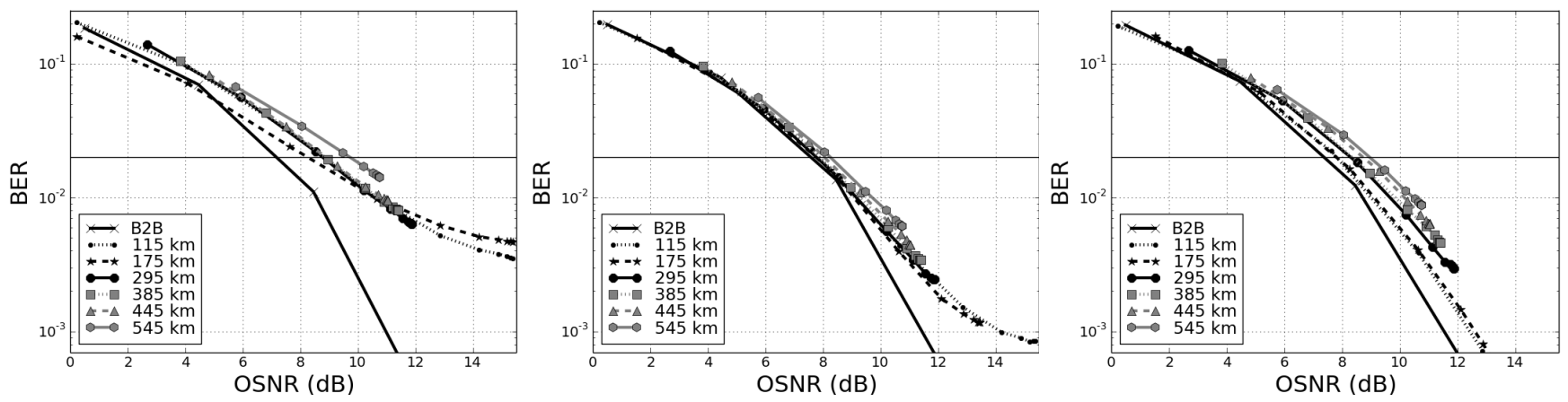

Fig. $8 \mathrm{BER}$ performance at 10Gb/s for different paths of the first, third and fifth band (from left to right, respectively). 
consequence, the BER performance varies depending on the OFDM band number and the considered path.

In Fig. 8, the BER performance of the first, third and last bands are shown, respectively. It can be observed that the results are below the target $\mathrm{BER}$ of $2 \cdot 10^{-2}$ (FEC threshold) for OSNR values ranging between $7 \mathrm{~dB}$ and $10 \mathrm{~dB}$. Successful transmission is obtained for all the bands and all the paths including the worst case. However, at the increase of the number of hops the influence of nonlinearities becomes more relevant leading to performance degradation. As expected, the first band is more affected by these effects. If a hard-decision FEC (HD-FEC) is considered with a target BER of $10^{-3}$, the bandwidth occupancy slightly decreases due to the reduced overhead (7\%). However, in none of the considered paths $10 \mathrm{~Gb} / \mathrm{s}$ transmission can be supported by the first band. The third and fifth bands are less affected by nonlinearities, showing performance below the HD-FEC limit in case of few hops paths, as can be argued from Fig. 8.

\section{Cost-effective BVT}

To further simplify the data plane solution and make it cost-effective, low complexity DSP can be adopted. This is achieved by performing the OFDM modulation with a realvalued transform as the Fast Hartley transform (FHT) or either implementing the real-valued FFT [15]. Fig. 9a and Fig. 9b show the DSP blocks required for these two schemes.

In [7], a cost-effective BVT design based on OFDM adopting the FHT processing has been numerically and experimentally assessed. It has been proposed as a building block for the S-BVT at the BRAS node. Bit loading schemes and guard band minimization have been analyzed for distance adaptive transmission, considering up to $10 \mathrm{~Gb} / \mathrm{s}$ connections and $12.5 \mathrm{GHz}$ channels.

However, to provide higher capacity and flexibility towards a centralized BRAS scenario, enabling more robust transmission over longer optical paths (e.g. to cover the calculated worst path), enhanced DSP is required. As shown in previous Sec. IV.A and IV.B, complex FFT with SSB modulation in combination with subcarrier multiplexing allows serving several MTUs in the regional network, with a limited number of optoelectronic blocks. Further optimized algorithms for bit and power loading could be suitably included for performance enhancement in terms of spectral efficiency, achievable distance and flexibility. Indeed, these improvements are at the expenses of increasing the S-BVT cost and complexity. This issue is particularly relevant for the transceiver design at the MTU side, where a very simple and cost-effective BVT is highly desirable. To this extent, for the BVT located at the MTU switch, we propose to adopt a simplified variant of the OFDM modulation, namely discrete multitone (DMT). The BVT scheme and the baseband processing are shown in Fig. 9. Nevertheless, CD can severely affect DMT systems, introducing power fading effect and reducing the maximum achievable reach.

To further lower the cost and complexity of the proposed solution, the use of direct modulated laser (DML) could be envisioned, also facilitating a higher integration density if the transceiver would be implemented in photonic integrated circuit technology. Nevertheless, challenging issues (such as chirp management and tune-ability range) should be taken into account and carefully addressed in

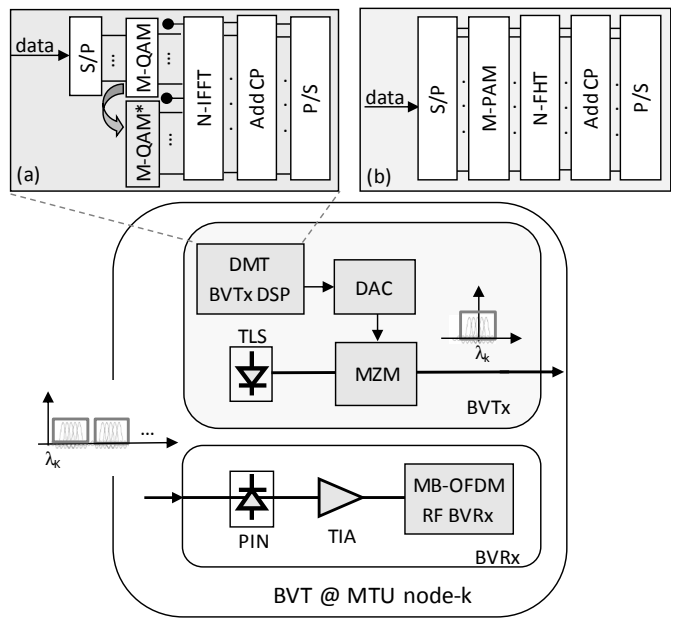

Fig. 9 BVT sited at the $k$-th MTU node. The DSP can be based either on (a) real-valued FFT or (b) FHT.

order to target the identified requirements and link lengths.

In order to cover the topology requirements of hundreds of $\mathrm{km}$, as defined in our study in Sec. III.B, an asymmetric approach for the downlink and uplink communication between BRAS and MTUs is envisioned [16].

We propose to keep simpler the BVTs at the multiple MTUs by combining amplitude modulated (AM) DMT, for the uplink transmission, with shared intradyne OFDM reception featuring phase diversity at the S-BVRx sited at the BRAS. As shown in Fig. 10, the AM signal is recovered at the BRAS receiver by means of a phase diversity frontend, enabling the detection of the full optical field. As shown in Fig. 11, a simplified DSP with lower computational complexity based on FHT or real-valued FFT is required to decode the MTU DMT signal. [15]. For the downstream communication, MB-OFDM with DD is adopted, as detailed in Sec. IV.A and experimentally validated in [10]. Similar to the connections between the BRAS and the MTUs, also in the proposed architecture for the uplink communication, different MTUs can be served by a single optoelectronic block at the BRAS S-BVRx. In fact, closely spaced MTU channels can be received by using a single local oscillator at the BRAS AM-OFDM receiver and further digitally processed. Thus, a preliminary digital RF down-conversion step per each MTU connection is required. Then, multiple

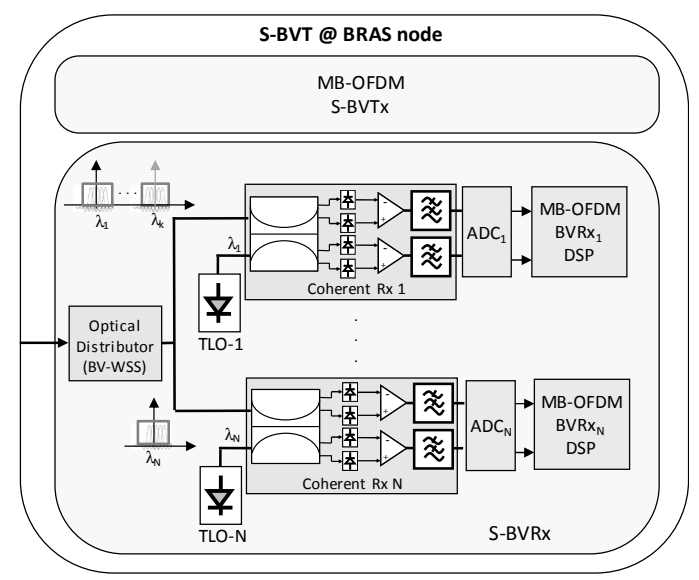

Fig. 10 S-BVT at BRAS node adopting AM-OFDM receiver array. 


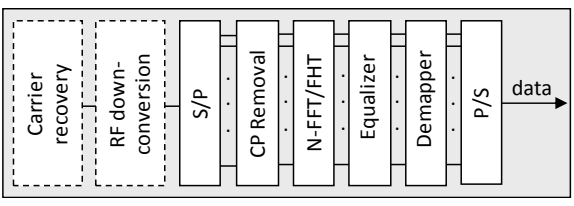

Fig. 11 (S)-BVRx DSP for a single band.

parallel DSP modules are used for detecting multiple MTU connections sharing the same optoelectronic block. Thus, the cost of a complex intradyne OFDM receiver can be shared among several MTUs.

We have experimentally assessed the transmission of $10 \mathrm{~Gb} / \mathrm{s}$ net connections from the MTUs to the virtual BRASes [16]. Here, we evaluate the performance in the ADREANALINE network of the same connections experimentally analyzed for the downstream (see [10], Sec. IV.A and Fig. 4). We consider uniform 4-QAM loading for a net $10 \mathrm{~Gb} / \mathrm{s}$ connection. The overhead consists of $7 \% \mathrm{FEC}$, for a $10^{-3}$ target BER, and $13 \%$, due to TS (4 over 64 frames), $\mathrm{CP}(5 \%)$ and a minimum guard band (4 DMT subcarriers over 256) for synchronization purposes. This results in a total electrical bandwidth of $6.1 \mathrm{GHz}$, including $21 \%$ total overhead. In the experimental set-up, the digital DMT signal is generated offline using Python code and converted to the analog domain by means of an arbitrary waveform generator running at $20 \mathrm{GSa} / \mathrm{s}$. The optical DMT signal is generated by using a MZM driven by an external cavity laser with $100 \mathrm{kHz}$ linewidth and centered at $1550.12 \mathrm{~nm}$. The experimental validation is performed in back-to-back and over $85 \mathrm{~km}$ and $185 \mathrm{~km} \mathrm{2-hop} \mathrm{paths} \mathrm{of} \mathrm{the} \mathrm{4-node}$ ADRENALINE mesh network. No inline dispersion compensation is adopted and the signals are received at the same node (BRAS node) by an S-BVT using intradyne detection. An optical filter is used (as WSS) to select the portion of the spectrum to be detected. The receiver uses, as tunable local oscillator (TLO), the same optical laser source at the related transmitter. The combined output of the $90^{\circ}$ optical hybrid is photodetected by a couple of balanced detectors and further acquired by a real-time oscilloscope running at $100 \mathrm{GSa} / \mathrm{s}$. The offline DSP consists of a first module for carrier recovery, followed by DMT demodulation, one-tap equalization and demapping. Results are shown in Fig. 12 for back-to-back and the proposed paths. At $10^{-3}$

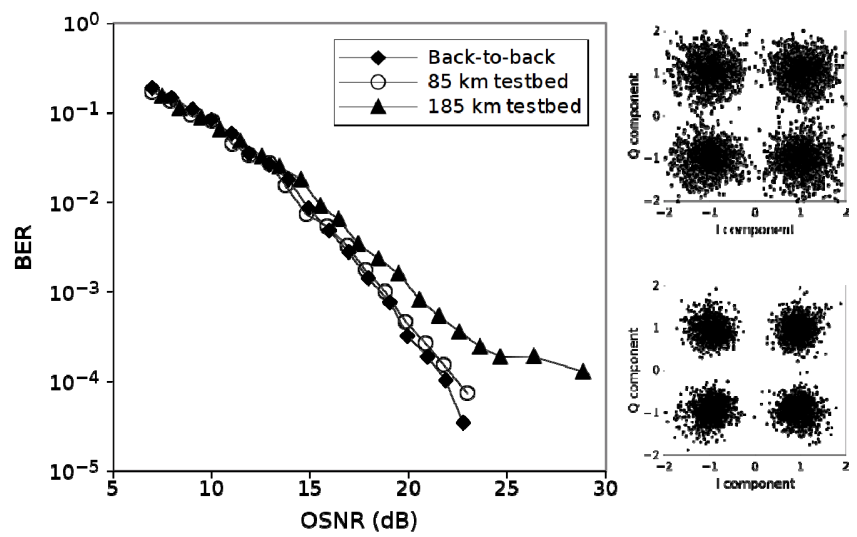

Fig. 12 BER vs OSNR performance of MTU-BRAS connections at $10 \mathrm{~Gb} / \mathrm{s}$ (left); received constellations after $85 \mathrm{~km}$ path corresponding to BER values of $10^{-3}$ (top right) and $10^{-4}$ (bottom right).
$\mathrm{BER}$, the required OSNR for the $85 \mathrm{~km}$ path is $18.8 \mathrm{~dB}$, $0.1 \mathrm{~dB}$ away from the back-to-back result. The path of $185 \mathrm{~km}$ requires an OSNR of $20.3 \mathrm{~dB}, 1.6 \mathrm{~dB}$ away from the back-to-back case. Also the difference between the required OSNR for the two paths is only $1.5 \mathrm{~dB}$.

\section{TECHNO-ECONOMIC ANALYSIS}

To compare the two alternative network scenarios presented in Fig 1a and Fig 1b we performed a technoeconomic analysis. In particular, we studied scenarios based on: (i) fixed-grid WDM technology and (ii) flexgrid technology, under two (S)-BVT variations: (ii.a) with coherent (CO) 100Gb/s technology and (ii.b) with MB-OFDM adopting DD. We calculate the cost of the first scenario (WDM) and use that as reference to calculate the target costs of components used in the flexgrid scenarios to achieve $30 \%$ cost savings, judged to be significant enough savings to motivate the adoption of the new flex-grid technology. In the evaluation, we take into account $1+1$ protection for both fixed-grid WDM and flexgrid scenarios. The cost calculations are based on the cost model presented in [17] (an extension of [18]).

\section{Scenario i- WDM solution:}

The optical network consists of WDM ROADMs and the traffic from the MTU switches is aggregated at a second level of aggregation switches before it enters the WDM network. Taking the reference TID Region-A domain, we have 200 MTU switches and 62 aggregation switches. Each MTU switch forwards $C=10 \mathrm{~Gb} / \mathrm{s}$ and is connected to two different aggregation switches for protection. Assuming equal distribution of load to the aggregation switches, each switch needs in total $14 \times 10 \mathrm{~Gb} / \mathrm{s}$ ports (for down- and uplink, including protection ports). We also assume that the communication between the MTUs and aggregation switches is done with $10 \mathrm{~Gb} / \mathrm{s}$ grey-short reach transceivers and the communication between the aggregation switches and the BRAS servers is done with $10 \mathrm{~Gb} / \mathrm{s}$ colored transceivers (routed over the WDM regional network). In the cost calculation we add the costs of (i) the MTU switches' transceivers (grey - short reach), (ii) the aggregation switches $(140 \mathrm{~Gb} / \mathrm{s}$ capacity, grey transceivers facing the MTUs and colored transceivers facing the WDM network), and (iii) transceivers (colored) at the BRASes. According to the cost model, the total cost of the WDM case is 219.82 ICU (IDEALIST Cost Unit, defined in [17]).

\section{Scenario ii-Flexgrid solution:}

We now assume that we replace the WDM network with a flexgrid network, and thus we include in the cost calculation the cost of the flexgrid WSS. We examine two variations for the transceivers: (ii.a) $\mathrm{CO}$ and (ii.b) MB-OFDM.

For case (ii.a) we assume the use of $10 \mathrm{~Gb} / \mathrm{s}$ grey - short reach transceivers at the MTUs and coherent $100 \mathrm{~Gb} / \mathrm{s}$ flexgrid BVTs (10x10Gb/s muxponders) at the add/drop ports of the flexgrid regional network, and coherent $400 \mathrm{~Gb} / \mathrm{s}$ S-BVT at the BRAS servers (functioning as $4 \times 100 \mathrm{~Gb} / \mathrm{s}$ ). We assume that each ROADM is equally loaded, so it serves 14 MTU switches (including connections for protection). Thus, each ROADM utilizes 2 flexgrid BVTs. On the BRAS nodes, one $400 \mathrm{~Gb} / \mathrm{s}$ S-BVT (with cost $X_{400 G-S B V T}$ ) serves two 
different ROADMs, which means that in total we need $15 \mathrm{~S}$ BVTs. To obtain $30 \%$ savings over the WDM case we calculate that we need $X_{400 G-S B V T} \leq 0.01 \mathrm{ICU}$, which is impossible to achieve.

For case (ii.b) we assume $10 \mathrm{~Gb} / \mathrm{s}$ MB-OFDM transceivers using DD at the MTUs and the MB-OFDM S-BVT proposed in Sec. IV.A at the BRAS (supporting $N=8$ flows, each flow serving $M=5$ MTUs at $10 \mathrm{~Gb} / \mathrm{s}$, as in Sec. IV.B). As above, we assume that each ROADM is equally loaded, so it serves 14 MTUs. Thus, each ROADM needs 3 flows, and in total we need 10 MB-OFDM S-BVTs (with cost $X_{\text {MB-OFDM-SBVT). To }}$ achieve cost savings of $30 \%$ over the WDM network, we find that $X_{M B-O F D M-S B V T} \leq 2.9 \mathrm{ICU}$, which is quite promising, since a same rate coherent S-BVT is considered to cost around 3 ICU in 2015 [17], and the cost of the proposed S-BVT would be much lower.

\section{Conclusion}

We examined the suitability of using flexgrid technologies for an evolutionary MAN scenario, where the photonic mesh network is expanded to the regional area and the aggregation network is extended in order to centralize the BRAS functionalities. A network architecture using flexgrid technology was proposed, leveraging the finer spectrum granularity to remove the second aggregation level in the regional network scenario. This solution exploits also the advanced features of S-BVT, envisioned to be used at the BRAS servers. After defining the network design problem and providing appropriate algorithmic solutions, we identified the related requirements for the region-A of Telefonica Spanish network.

Driven by those requirements, we then presented suitable data plane solutions, targeting cost reduction and robustness to transmission impairments. We proposed an SBVT architecture based on MB-OFDM to reduce the number of optoelectronic components and support the multiple low bit rate connections between the MTUs and the BRAS pool over regional optical paths. We demonstrated that adopting cost-effective DD, up to 5 bands $(50 \mathrm{~Gb} / \mathrm{s})$ per flow can be successfully transmitted, which is suitable for serving the multiple endpoints according to the topology requirements. In addition, an asymmetric architecture for the connectivity between the MTUs and BRAS was proposed to further simplify the MTU transceiver at a cost of introducing more complexity in the shared S-BVT receiver at the BRAS.

Finally, the flexgrid regional network architecture was compared to WDM from a techno-economic point of view, showing that the coherent flexgrid transponders envisioned for core networks are expensive, while the proposed transponders using MB-OFDM and DD are a viable solution for the application of flexgrid technology in the MAN.

\section{ACKNOWLEDGMENT}

Work partially supported by the EU-FP7 IDEALIST project (G. A. 317999), the Spanish MINECO DESTELLO project (TEC2015-69256-R) and the FPI research scholarship grant BES-2013-064397.

\section{REFERENCES}

[1] M. Jinno, et al., "Spectrum-efficient and scalable elastic optical path network: architecture, benefits, and enabling technologies," IEEE Commun. Mag., vol. 47, no. 11, pp. 66-73, 2009.

[2] O. Gerstel, et al., "Elastic optical networking: a new dawn for the optical layer?," IEEE Commun. Mag., vol. 50, s12-s20, Feb. 2012.

[3] A. Napoli et al., "Next generation elastic optical networks: The vision of the European research project IDEALIST," IEEE Communications Magazine, vol. 53, pp. 152-162, Feb. 2015

[4] N. Sambo et al., "Next Generation Sliceable Bandwidth Variable Transponders," IEEE Commun. Mag., vol. 53, pp. 163-171, Feb. 2015

[5] M. Svaluto Moreolo, et. al., "SDN-enabled Sliceable BVT Based on Multicarrier Technology for Multi-Flow Rate/Distance and Grid Adaptation," IEEE/OSA J. Lightwave Technol., vol. 34, no. 6, pp. 1516-1522, March 2016.

[6] "ITU-T Recommendation G.694.1, Spectral grids for WDM applications: DWDM frequency grid.” Feb. 2012.

[7] M. Svaluto Moreolo, et al., "Cost-Effective Data Plane Solutions Based on OFDM Technology for Flexi-Grid Metro Networks Using Sliceable Bandwidth Variable Transponders," In Proc. ONDM, 2014.

[8] Network Function Virtualization White Paper https://portal.etsi.org/Portals/0/TBpages/NFV/Docs/NFV_White _Paper3.pdf

[9] W. Shieh and I. Djordjevic, OFDM for Optical Communications, USA, Elsevier, 2010.

[10] M. Svaluto Moreolo et al., "Assessment of Flexgrid Technologies in the MAN for Centralized BRAS Architecture Using S-BVT," In Proc. ECOC, 2014, paper P.6.9.

[11] S. L. Jansen, I. Morita, T. C. W. Schenk, H. Tanaka, "121.9Gb/s PDM-OFDM Transmission with $2-\mathrm{b} / \mathrm{s} / \mathrm{Hz}$ Spectral Efficiency Over $1000 \mathrm{~km}$ of SSMF," in IEEE/OSA J. Lightwave Technol., vol.27, no.3, pp.177-188, Feb. 2009

[12] W. R. Peng, I. Morita, H. Takahashi, T. Tsuritani, "Transmission of High-Speed ( $>10 \mathrm{~Gb} / \mathrm{s})$ Direct-Detection Optical OFDM Superchannel,", IEEE/OSA J. Lightwave Technol., vol.30, no.12, pp.2025-2034, Jun. 2012

[13] L. Nadal, M. Svaluto Moreolo, J.M. Fàbrega, G. Junyent, "Low Complexity PAPR Reduction Techniques for Clipping and Quantization Noise Mitigation in Direct-Detection O-OFDM Systems," Optical Fiber Technol., vol. 20, pp. 208-216, 2014.

[14] L. Nadal, M. Svaluto Moreolo, J. M. Fabrega, A. Dochhan, H. Griesser, M. Eiselt, and J. P. Elbers, "DMT modulation with adaptive loading for high bit rate transmission over directly detected optical channels," IEEE/OSA J. Lightwave Technol., vol. 32, no. 21, pp. 3541-3551, Nov. 2014.

[15] M. Svaluto Moreolo, R. Muñoz, and G. Junyent, "Novel power efficient optical OFDM based on Hartley transform for intensity-modulated direct-detection systems," IEEE/OSA J. Lightwave Technol. 28, 798-805 (2010).

[16] J. M. Fàbrega, M. Svaluto Moreolo, F. J. Vílchez, K. Christodoulopoulos, E. Varvarigos, J. P. Fernández-Palacios, "Experimental validation of MTU-BRAS connectivity with DMT transmission and coherent detection in flexgrid metro networks using sliceable transceivers," In Proc. OFC 2015, Th3H.4.

[17] Idealist deliverable D1.1: "Elastic Optical Network Architecture: reference scenario, cost and planning".

[18] F. Rambach, B. Konrad, L. Dembeck, U. Gebhard, M. Gunkel, M. Quagliotti, L. Serra, and V. López, "A Multilayer Cost Model for Metro/Core Networks," J. Optical Communications and Networking, Vol. 5 (3), pp. 210-225, 2013.

[19] M. Andrews, L. Zhang, "Complexity of wavelength assignment in optical network optimization", IEEE Infocom 2006.

[20] R. Bhandari, "Survivable Networks: Algorithms for Diverse Routing”, Springer, 1999. 